\title{
Real-time PCR genotyping assay for feline erythrocyte pyruvate kinase deficiency and mutant allele frequency in purebred cats in Japan
}

\author{
Kazuya KUSHIDA ${ }^{1)}$, Urs GIGER ${ }^{2)}$, Toshihiko TSUTSUI ${ }^{3)}$, Megumi INABA ${ }^{3)}$, Yoshio KONNO ${ }^{3)}$, Kureha HAYASHI ${ }^{3)}$, \\ Kana NOGUCHI ${ }^{3)}$, Akira YABUKI ${ }^{1)}$, Keijiro MIZUKAMI ${ }^{1,2)}$, Moeko KOHYAMA ${ }^{1)}$, Yasuyuki ENDO ${ }^{4)}$ and \\ Osamu YAMATO ${ }^{1) *}$ \\ 1) Laboratory of Clinical Pathology, Joint Faculty of Veterinary Medicine, Kagoshima University, 1-21-24 Kohrimoto, Kagoshima \\ 890-0065, Japan \\ ${ }^{2)}$ Section of Medical Genetics, School of Veterinary Medicine, University of Pennsylvania, Philadelphia, PA 19104, U.S.A. \\ 3) International Institute of Small Animal Medicine (Bio Plus), AHB Inc., 3-7-11 Kiba, Koutou-ku, Tokyo 135-0042, Japan \\ 4)Laboratory of Small Animal Internal Medicine, Joint Faculty of Veterinary Medicine, Kagoshima University, 1-21-24 Kohrimoto, \\ Kagoshima 890-0065, Japan
}

(Received 17 November 2014/Accepted 2 February 2015/Published online in J-STAGE 13 February 2015)

ABSTRACT. Erythrocyte pyruvate kinase (PK) deficiency is an inherited glycolytic erythroenzymopathy caused by mutations of the $P K L R$ gene. A causative mutation of the feline PKLR gene was originally identified in Abyssinian and Somali cats in the U.S.A. In the present study, a TaqMan probe-based real-time PCR genotyping assay was developed and evaluated for rapid genotyping and large-scale screening for this mutation. Furthermore, a genotyping survey was carried out in a population of four popular purebred cats in Japan to determine the current mutant allele frequency. The assay clearly displayed all genotypes of feline PK deficiency, indicating its suitability for large-scale survey as well as diagnosis. The survey demonstrated that the mutant allele frequency in Abyssinian and Somali cats was high enough to warrant measures to control and prevent the disease. The mutant allele frequency was relatively low in Bengal and American Shorthair cats; however, the testing should still be carried out to prevent the spread of the disease. In addition, PK deficiency should always be considered in the differential diagnosis of anemia in purebred cats in Japan as well as worldwide.

KEY WORDS: anemia, feline PKLR gene, mutant allele frequency, pyruvate kinase deficiency, real-time PCR genotyping

doi: 10.1292/jvms.14-0600; J. Vet. Med. Sci. 77(6): 743-746, 2015

Pyruvate kinase (PK, EC2.7.1.40) is a glycolytic enzyme that catalyzes transphosphorylation from phosphoenolpyruvate to ADP, yielding pyruvate and ATP $[3,7]$. It is the last step of the glycolytic pathway and is essentially irreversible. Erythrocyte PK deficiency, an autosomal recessive inherited glycolytic erythroenzymopathy resulting in a chronic hemolytic anemia, was first documented in humans in the early 1960s [16] (Online Mendelian Inheritance in Man [13], OMIM 266200), in dogs in the 1970s [14] and cats in the early 1990s [2, 4] (Online Mendelian Inheritance in Animals [12], OMIA 000844-9615 and 9685, respectively). This disease is caused by mutations of the PKLR gene encoding the liver and red blood cell PK isozymes [3, 12, 13].

Feline PK deficiency was originally identified in Abyssinian and Somali cats in the U.S.A. in the early 1990s [2, 4, 12]. In contrast to affected dogs and humans, PK deficient cats have an intermittent rather than chronic hemolytic anemia. Bilirubin gall bladder stones and posthepatic obstruction are also characteristics in PK deficient cats. A causative

*Correspondence to: Yamato, O., Laboratory of Clinical Pathology, Joint Faculty of Veterinary Medicine, Kagoshima University, 1-21-24 Kohrimoto, Kagoshima 890-0065, Japan.

e-mail: osam@vet.kagoshima-u.ac.jp

(C)2015 The Japanese Society of Veterinary Science

This is an open-access article distributed under the terms of the Creative Commons Attribution Non-Commercial No Derivatives (by-nc-nd) License $<$ http://creativecommons.org/licenses/by-nc-nd/3.0/>. mutation of the feline PKLR gene was detected in 1997 [4], while five mutations have been described in dogs $[6,15,17]$ and approximately 240 DNA variants and/or mutations in humans [10]. The mutation causing feline PK deficiency is a deletion of the last 13 bases of exon 5 from mRNA due to a splicing defect, which is consequently correlated with a single nucleotide substitution mutation (c.693+304G $>$ A) of intron 5 in genomic DNA [4, 5, 12]. Although expression studies have not yet been done, it seems likely that with varied splicing, the normal and mutant alleles may be expressed in different quantities which could explain the intermittent signs.

A few studies have reported that the frequency of mutant allele for PK deficiency in Abyssinian and Somali cats is high in the U.S.A., Europe and Australia [1, 5, 8]. Furthermore, this mutation has been found in many purebred cats other than Abyssinian and Somali cats in the U.S.A. and Europe and even domestic shorthair cats [5]. However, there has been no information describing the mutant allele frequency of feline PK deficiency in Japan. In addition, an easy and reliable genotyping method is required for diagnosis and screening for this disease.

In the present study, a TaqMan probe-based real-time PCR genotyping assay was developed and evaluated for rapid genotyping and large-scale screening for the mutation of feline PK deficiency. Furthermore, a genotyping survey was carried out in a population of four popular purebred cats in Japan to determine the current mutant allele frequency. 
Table 1. Primers and probes used in real-time PCR assay for rapid genotyping and large-scale screening for the mutation of feline pyruvate kinase deficiency

\begin{tabular}{llccc}
\hline \multicolumn{1}{c}{ Primer/probe } & \multicolumn{1}{c}{ Sequence $5^{\prime} \rightarrow 3^{\prime}(\mathrm{mer})$} & Reporter $\left(5^{\prime}\right)$ & Quencher $\left(3^{\prime}\right)$ & Concentration $(\mathrm{nM})$ \\
\hline Forward primer & GACACGGGTTCCTGATTTCCT $(21)$ & NA & NA & 450 \\
Reverse primer & CCTGCAGGCATGGGAAGAG $(19)$ & NA & NA & 450 \\
Probe for wild-type allele & CCACGACTCTGCC $(13)$ & FAM & NFQ & 100 \\
Probe for mutant allele & CTCCACAACTCTGCC $(15)$ & VIC & NFQ & 100 \\
\hline
\end{tabular}

$\mathrm{NA}=$ not applicable; $\mathrm{FAM}=6$-carboxyfluorescein; $\mathrm{VIC}=6$-carboxyrhodamine; $\mathrm{NFQ}=$ nonfluorescent quencher. Underlines letter in the sequence of the probe indicates adenine corresponding to a substitution mutation $(c .693+304 \mathrm{G}>\mathrm{A})$ in feline pyruvate kinase deficiency.

All experimental procedures involving animals and their samples were performed in accordance with the guidelines regulating animal use at Kagoshima University.

Control DNA samples from affected and carrier cats maintained at the University of Pennsylvania and wild-type healthy cats in Japan were used to evaluate the genotyping assay after the genotypes were confirmed by direct sequencing. In addition, whole blood samples were collected from 35 Abyssinian, 48 Somali, 63 Bengal and 59 American Shorthair cats for the survey. The samples of Abyssinian, Somali and Bengal cats were collected from 29 contract catteries $(8,13$ and 12 catteries, respectively) of a single pet dealer (AHB Inc., Tokyo, Japan). These catteries had separate breeding programs and were scattered throughout Japan. The samples of American Shorthair cats were randomly collected from several animal hospitals with the consent of owners. The whole blood samples $(0.1-0.3 \mathrm{~m} l)$ were spotted onto a Flinders Technology Associates filter paper (FTA card; Whatman International Ltd., Piscataway, NJ, U.S.A.) for storage.

For the real-time PCR assay, primers and TaqMan minor groove binder (MGB) probes listed in Table 1 were designed based on the sequences of the feline PKLR gene in a wildtype cat and a cat affected with PK deficiency (GenBank accession numbers XM_006943090.1 and JX951425.1). These primers and probes, bound each with a fluorescent reporter dye (6-carboxyrhodamine or 6-carboxyfluorescein) at the $5^{\prime}$-end and a nonfluorescent quencher dye at the $3^{\prime}$-end, were synthesized by a commercial company (Applied Biosystems, Foster City, CA, U.S.A.). For the DNA template, a disc punched out of the FTA cards was used for DNA extraction as previously described [11]. The amplifications were carried out using the OneStep real-time PCR system (Applied Biosystems) in a final volume of $10 \mu l$ consisting of $2 \times$ PCR master mix (TaqMan GTXpress Master Mix, Applied Biosystems), 80× genotyping assay mix (TaqMan SNP Genotyping Assays, Applied Biosystems) containing the specific primers, TaqMan MGB probes (Table 1) and template DNA. Before PCR, the prepared PCR mixtures were held at $25^{\circ} \mathrm{C}$ for $30 \mathrm{sec}$. The cycling conditions consisted of $20 \mathrm{sec}$ at $95^{\circ} \mathrm{C}$ followed by 50 cycles of $3 \mathrm{sec}$ at $95^{\circ} \mathrm{C}$ and $20 \mathrm{sec}$ at $60^{\circ} \mathrm{C}$. The holding stage after PCR was carried out at $25^{\circ} \mathrm{C}$ for $30 \mathrm{sec}$. The obtained data were analyzed with StepOne software version 2.3 (Applied Biosystems), and the calculations were based on the results obtained using DNA samples from control cats.
Pedigree analysis was performed for Abyssinian, Somali and Bengal cats to elucidate the genetic relationship of affected and carrier cats identified in Japan. The genetic relationships were analyzed using pedigree information by the pet dealer and a few pedigree databases. Pedigree analysis was not performed in American Shorthair cats, because pedigree information was not available.

The real-time PCR assay clearly identified all the genotypes according to three patterns in the amplification of the wild-type and/or mutant alleles (Fig. 1). The total required time for 50-cycle amplification was within $50 \mathrm{~min}$. Although very minor nonspecific allelic amplification was observed in the homozygous mutant genotype in which false wild-typeallelic amplification was slightly increased together with true mutant-allelic amplification because of an incomplete specificity in the probe for the mutant allele, it was possible to distinguish between the allelic types clearly.

The results of the survey among four popular purebred cats in Japan are shown in Table 2. The mutant allele was present in all breeds examined in this study. The mutant allele frequency was high in Abyssinian (0.214) and Somali cats (0.229) and relatively low in Bengal (0.016) and American Shorthair cats $(0.008)$. The mutant allele frequency of the cohort of the four breeds together was 0.098. In this survey, two affected kittens were identified, but could not be followed up clinically and hematologically.

The pedigree analysis demonstrated some relationships between cats of the same breed, but not all carrier and affected cats in each breed were closely related with one another in Abyssinian and Somali cats (data not shown). These cats were widely distributed in different parts of Japan and among many families in these two breeds. However, two Bengal carriers identified in the present survey had a common grandsire (data not shown).

In the present study, a real-time PCR assay using TaqMan MGB probes was developed to provide clear-cut genotyping results for the mutant and wild-type alleles to detect in PK deficiency in cats (Fig. 1). The required time for the PCR was short (about $50 \mathrm{~min}$ ). The use of FTA cards for sampling eliminated the need for traditional multistep extraction and purification procedures [11], allowing for early reporting of the results within $2 \mathrm{hr}$ after sample collection in combination with the real-time PCR technique. The results in the present study showed that the assay is a simple, rapid and reliable tool for large-scale epidemiological surveys as well as a diagnostic method for feline PK deficiency. 
Table 2. The number of cats (carrier and affected) with genotypes for pyruvate kinase deficiency and allele frequency for the feline $P K L R$ mutation in various cat breeds

\begin{tabular}{lcccc}
\hline \multicolumn{1}{c}{ Cat breed } & $\begin{array}{c}\text { Number of } \\
\text { examined cats }\end{array}$ & $\begin{array}{c}\text { Number of } \\
\text { carriers (\%) }\end{array}$ & $\begin{array}{c}\text { Number of } \\
\text { affected cats (\%) }\end{array}$ & $\begin{array}{c}\text { Mutant allele } \\
\text { frequency }\end{array}$ \\
\hline Abyssinian & 35 & $13(37.1)$ & $1(2.9)$ & 0.214 \\
Somali & 48 & $20(41.7)$ & $1(2.1)$ & 0.229 \\
Bengal & 63 & $2(3.2)$ & $0(0)$ & 0.016 \\
American Shorthair & 59 & $1(1.7)$ & $0(0)$ & 0.008 \\
Total & 205 & $36(17.6)$ & $2(1.0)$ & 0.098 \\
\hline
\end{tabular}
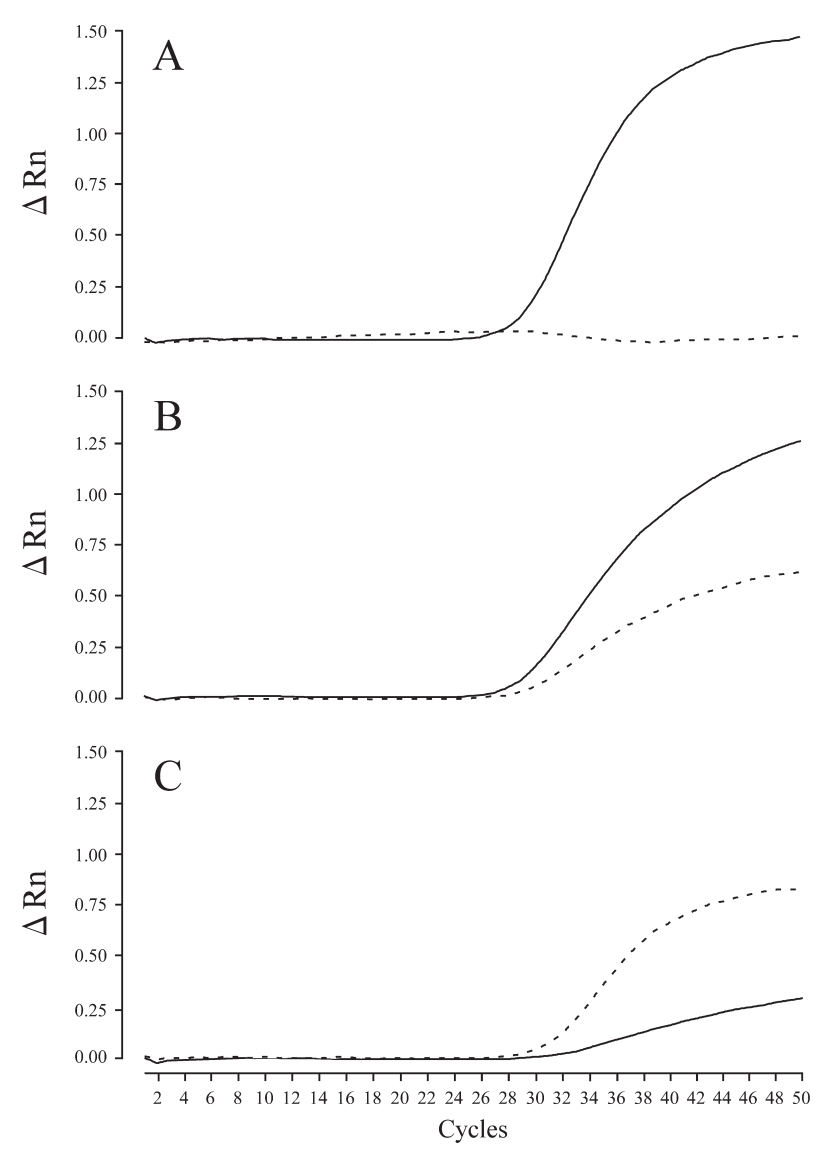

Fig. 1. Real-time PCR amplification plots of wild-type and mutant alleles in feline pyruvate kinase deficiency. Amplification was plotted as fluorescence intensity ( $\Delta \mathrm{Rn}$ value) against cycle number. The $\Delta \mathrm{Rn}$ value is the reporter dye signal normalized to the internal reference dye and corrected for the baseline signal established in the first few cycles of PCR. Each of the three amplification plots showed the wild-type (A), heterozygous carrier (B) and mutant genotypes (C). Solid and dotted lines indicate amplification in the presence of wild-type and mutant alleles, respectively.

The results of the survey in the present study demonstrated that the current mutant allele frequency in Abyssinian and Somali cats in Japan is similarly high compared to that in other countries (Table 3 ). The pedigree analysis indicated that the mutant allele of PK deficiency is distributed widely in the population of Abyssinian and Somali cats in Japan.
Table 3. The mutant allele frequency of feline PK deficiency in Japan and other countries

\begin{tabular}{lccccc}
\hline \multicolumn{1}{c}{ Cat breed } & Japan* & U.S.A.** & U.K.** & Germany & Australia \\
\hline Abyssinian & 0.214 & 0.126 & 0.111 & 0.276 & 0.125 \\
Somali & 0.229 & 0.085 & 0.138 & 0.205 & 0.292 \\
Bengal & 0.016 & 0.164 & 0.170 & ND & ND \\
American Shorthair & 0.008 & 0 & 0 & ND & ND \\
\hline
\end{tabular}

*Data reported in the present study. **Data reported in 2012 [5]. $\uparrow$ Data reported in 2005 [8]. ††Data reported in 2009 [1]. ND: not determined.

Therefore, the testing of Abyssinian and Somali cats for breeding is strongly recommended to reduce the mutant allele frequency and finally lead to the control and prevention of the disease in these two breeds. Screening prior to breeding can avoid any breeding of PK deficient cats and breeding between carriers and thereby prevent production of any affected cats in the future while preserving the gene pool in each breed.

Bengal cats are a hybrid of the Asian leopard cat and domestic cats, such as an Abyssinian cat, and was developed in the 1970s and 80s. In this survey, the mutant allele frequency was low in Bengal cats in Japan, which is about one-tenth of that in cats in the U.S.A. and Europe (Table 3 ). The pedigree analysis indicated that two related Bengal carriers identified in this survey were with a limited pedigree, suggesting that many of the Bengal cat pedigrees in Japan are currently free from the PK mutation. However, if cats carrying the mutation are imported from the U.S.A. or Europe, where the mutant frequency is much higher, the frequency in Japan may increase rapidly. In American Shorthair cats, the most popular breed in Japan, the mutant allele for PK deficiency was detected for the first time in the present study, although at a low frequency. Population stratification is the presence of a systematic difference in allele frequencies between subpopulations in a population and is mainly caused by nonrandom mating between groups, often due to their physical separation. In inherited diseases, population stratification in purebred animals can easily occur unknowingly in a geographical region and/or in a limited cattery by breeding animals with a mutant PK allele (i.e., founder effect by popular sires with a mutant allele), resulting in an elevation of the mutant allele frequency and disease prevalence in that region. Therefore, the testing of Bengal and American Shorthair cats intended for breeding is also recommended as a control and prevention of the disease. 
The study examining clinical signs, laboratory parameters and a course of disease in Abyssinian and Somali cats with PK deficiency demonstrated that this disease shows variation in age of onset and severity of clinical signs [9]. In addition, since many affected cats remain completely asymptomatic for years after birth, the associated anemia may not be the primary reason for a PK-deficient cat's illness [3]. These facts suggest that additional factors may be required to induce the disease, such as stress and activity level [9]. Other genetic backgrounds may be associated with variation in clinical course and features in PK deficient cats. In the present study, two affected kittens identified by the survey could not be followed up; therefore, there has been no feedback information toward the genotype-phenotype correlation. In the near future, the accumulated clinical follow-up data will help provide a better overall picture of the feline PK deficiency.

In conclusion, the present study demonstrated that the prevalence of PK deficiency varies in four popular purebred cats in Japan to warrant the measures for the control and prevention of the disease in Abyssinian and Somali cats and for the screening in Bengal and American Shorthair cats imported for breeding. In addition, PK deficiency should always be considered in the differential diagnosis of anemia in purebred cats.

ACKNOWLEDGMENTS. The authors are grateful to $\mathrm{Mr}$. Yutaka Okada of AHB Inc. for collecting samples. This study was supported by the Ministry of Education, Culture, Sports, Science and Technology of Japan (MEXT grant no. 25292181 to OY) and National Institutes of Health (NIH grant no. OD 010939 to UG).

\section{REFERENCES}

1. Barrs, V. R., Giger, U., Wilson, B., Chan, C. T., Lingard, A. E., Tran, L., Seng, A., Canfield, P. J. and Beatty, J. A. 2009. Erythrocytic pyruvate kinase deficiency and AB blood types in Australian Abyssinian and Somali cats. Aust. Vet. J. 87: 39-44. [Medline] [CrossRef]

2. Ford, S., Giger, U., Duesberg, C., Beutler, E. and Wang, P. 1992. Inherited erythrocyte pyruvate kinase (PK) deficiency causing hemolytic anemia in an Abyssinian cat. J. Vet. Intern. Med. 6: 123.

3. Giger, U. 2010. Hereditary erythrocyte enzyme abnormalities. pp. 179-186. In: Schalm's Veterinary Hematology, 6th ed. (Weiss, D. J. and Wardrop, K. J. eds.), Lippincott Williams and
Wilkins, Philadelphia.

4. Giger, U., Rajpurohit, Y., Wang, P., Ford, S., Kohn, B., Niggemeier, A., Patterson, D. F., Beutler, E. and Henthorn, P. S. 1997. Molecular basis of erythrocyte pyruvate kinase (R-PK) deficiency in cats. Blood 90 (Suppl): 5b. (abstract)

5. Grahn, R. A., Grahn, J. C., Penedo, M. C., Helps, C. R. and Lyons, L. A. 2012. Erythrocyte pyruvate kinase deficiency mutation identified in multiple breeds of domestic cats. BMC Vet. Res. 8: 207. [Medline] [CrossRef]

6. Gultekin, G. I., Raj, K., Foureman, P., Lehman, S., Manhart, K., Abdulmalik, O. and Giger, U. 2012. Erythrocytic pyruvate kinase mutations causing hemolytic anemia, osteosclerosis, and seconday hemochromatosis in dogs. J. Vet. Intern. Med. 26: 935-944. [Medline] [CrossRef]

7. Harvey, J. W. 2010. Erythrocyte biochemistry. pp. 131-135. In: Schalm's Veterinary Hematology, 6th ed. (Weiss, D. J. and Wardrop, K. J. eds.), Lippincott Williams and Wilkins, Philadelphia.

8. Kohn, B., Fumi, C., Seng, A. and Giger, U. 2005. Anemia due to erythrocytic pyruvate kinase deficiency and its incidence in Somali and Abyssinian cats in Germany. Kleintierpraxis 50: 305-312.

9. Kohn, B. and Fumi, C. 2008. Clinical course of pyruvate kinase deficiency in Abyssinian and Somali cats. J. Feline Med. Surg. 10: 145-153. [Medline] [CrossRef]

10. Leiden Open Variation Database (LOVD) http://www.lovd. n1/2.0/home Accessed April 9, 2015.

11. Mizukami, K., Chang, H. S., Yabuki, A., Kawamichi, T., Kawahara, N., Hayashi, D., Hossain, M. A., Rahman, M. M., Uddin, M. M. and Yamato, O. 2011. Novel rapid genotyping assays for neuronal ceroid lipofuscinosis in Border Collie dogs and high frequency of the mutant allele in Japan. J. Vet. Diagn. Invest. 23: 1131-1139. [Medline] [CrossRef]

12. Online Mendelian Inheritance in Animals http://omia.angis.org. au/home/ Accessed April 9, 2015.

13. Online Mendelian Inheritance in Man http://www.ncbi.nlm.nih. gov/omim/ Accessed April 9, 2015.

14. Searcy, G. P., Miller, D. R. and Tasker, J. B. 1971. Congenital hemolytic anemia in the Basenji dog due to erythrocyte pyruvate kinase deficiency. Can. J. Comp. Med. 35: 67-70. [Medline]

15. Skelly, B. J., Wallace, M., Rajpurohit, Y. R., Wang, P. and Giger, U. 1999. Identification of a 6 base pair insertion in West Highland White Terriers with erythrocyte pyruvate kinase deficiency. Am. J. Vet. Res. 60: 1169-1172. [Medline]

16. Valentine, W. N., Tanaka, K. R. and Miwa, S. 1961. A specific erythrocyte glycolytic enzyme defect (pyruvate kinase) in three subjects with congenital non-spherocytic hemolytic anemia. Trans. Assoc. Am. Physicians 74: 100-110. [Medline]

17. Whitney, K. M., Goodman, S. A., Bailey, E. M. and Lothrop, C. D. Jr. 1994. The molecular basis of canine pyruvate kinase deficiency. Exp. Hematol. 22: 866-874. [Medline] 